\title{
Muography of the Puy de Dôme
}

Pasquale Noli ${ }^{1{ }^{\star}}$, Fabio Ambrosino ${ }^{1,2}$, Lorenzo Bonechi ${ }^{3}$, Alan Bross $^{4}$, Luigi Cimmino ${ }^{1}$, Raffaello D’Alessandro ${ }^{3,5}$, Vincenzo Masone ${ }^{1}$, Nicola Mori ${ }^{3,5}$, Giuseppe Passeggio ${ }^{1}$, Anna Pla-Dalmau ${ }^{4}$, Giulio Saracino ${ }^{1,2}$, Enrico Scarlini ${ }^{3,4}$, Paolo Strolin ${ }^{1,2}$

\author{
${ }^{1}$ Istituto Nazionale di Fisica Nucleare (INFN), Sezione di Napoli, Naples, Italy \\ ${ }^{2}$ Università di Napoli Federico II, Dipartimento di Fisica, Naples, Italy \\ ${ }^{3}$ Istituto Nazionale di Fisica Nucleare (INFN), Sezione di Firenze, Sesto Fiorentino, Florence, Italy \\ ${ }^{4}$ Fermilab, Batavia, Illinois, USA \\ ${ }^{5}$ Università di Firenze, Dipartimento di Fisica e Astronomia, Sesto Fiorentino, Florence, Italy
}

\section{Article history}

Received July 5, 2016; accepted October 5, 2016.

Subject classification:

Detector, Muon radiography, Volcano, Cosmic rays, Muon.

\begin{abstract}
Muon radiography is an imaging technique that relies on the transmission of cosmic muons through matter. It allows the measurement of density maps of large structures such as volcanoes. During the second half of 2013 the MURAY detector prototype carried out a data taking at the Puy de Dôme in the framework of the scientific collaboration with the experiment TOMUVOL in order to compare the results and performance of the two different detectors. Both experimental apparatuses measure a muon transmission of some orders of magnitude higher than that expected highlighting a background that perturbs these measures.
\end{abstract}

\section{Introduction}

The Puy de Dôme is a large lava dome, originating from one of the youngest volcanoes in the region of Massif Central in south-central France. A campaign of muographic measurements was performed jointly by the MURAY [Ambrosino et al. 2014] and TOMUVOL [Cârloganu et al. 2013] collaborations, using different detector techniques. TOMUVOL (TOmographie MUonique des VOLcans) has been using resistive plate chambers (RPCs). MURAY (MUon RAdiographY) has been using an early prototype of a muon detector of a new generation, based on the use of plastic scintillators. The prototype was still incomplete for optimal background rejection. The purpose of this approach was a better understanding of systematic effects and of backgrounds, by a combined data analysis.

The MURAY detector was installed at the Col de Ceissat, in a location at $1078 \mathrm{~m}$ a.s.1. altitude. The data taking started at the end of June 2013 for about 92 days. We report the detector performances during the data taking and preliminary results, in particular concerning the background.

\section{Imaging with muons}

Muography is an imaging technique that enables to study the interior of large structures such as volcanoes, mountains or mines through the study of absorption of high energy muons created by the interactions of primary cosmic rays in the upper Earth's atmosphere. The absorption depends on the thickness and the density of the traversed rock. Its measurement provides data to construct a map of the average density of the crossed rock. Muography can directly image the inner structure of volcanoes in order to show the possible presence of lava conduits, dikes or zones with different densities.

Conventional measurement methods (gravimetric, seismological or electromagnetic) achieve resolutions of the order of several $100 \mathrm{~m}$. In optimal acquisition conditions, muography can improve the resolution by one order of magnitude, though limited to the volcano edifice. Moreover, combined with other techniques it enriches the information with a sheer density measurement that can help in solving ambiguities and disentangling the structural information.

The first application of the cosmic-ray muon flux to detect density variations in a large structure dates back almost 40 years and it is due to Luis Alvarez [Alvarez et al. 1970]. He investigated the internal structure of the pyramid of the Chefren Pharaon, looking for an unknown burial chamber. Indeed, unlike in the pyramids 
of his father Cheope and of his grandfather Sneferu, in the Chefren pyramid only one burial chamber has been found suggesting the existence of a hidden chamber. Using a muon detector based on the spark chamber technique, Alvarez and collaborators measured the directional cosmic ray flux penetrating through the pyramid. The technique was proven to be successful, although no evidence of a hidden chamber was found.

New technologies were needed for the development of detectors suitable for more extensive applications of muography. Since 2003, muography is being applied to volcanoes in Japan, France and Italy. The breakthrough occurred in 2007, almost forty years after Alvarez, providing an image of internal structure of the summit of Mt. Asama in Japan [Tanaka et al. 2007]. So far, muon radiographies have been performed in Japan over rock thicknesses of less than $1 \mathrm{~km}$. For a substantial progress in the practical application of muography, the sensitivity must be improved one or two orders of magnitude. To that purpose, the most critical item is background rejection. Muography implies a multi-disciplinary effort, as the basic detection techniques come from particle physics.

\section{The MURAY detector}

The MURAY muon telescope is based on the use of plastic scintillators bars. Volcanic areas are usually characterized by a hard environment and by difficulties of access, requiring a modular structure to ease transportation and installation. The basic "module" consists of a planar array of 32 plastic scintillator bars ( $1 \mathrm{~m}$ long) (Figure 1) with triangular cross-section and a central hole to allocate a wave-length shifting (WLS) fibre (Figure 2).

The triangular cross-section allows the construction of very compact, crack-free planar arrays. Moreover, the weighted average of the light output produced by two adjacent bars enables improving the spatial resolution.

The scintillator bars - similar to those used in the D0 [Baringer et al. 2001] and Minerva (http:/ / minerva. fnal.gov) experiments - have been produced by extrusion at the Fermilab NICADD facility. The scintillator is doped with blue-emitting fluorescent compounds (PPO $1 \%$ and POPOP $0.03 \%$ ). The central hole is co-extruded, together with a $0.25 \mathrm{~mm}$ thick titanium oxide coating that increases the internal reflectivity and shields from

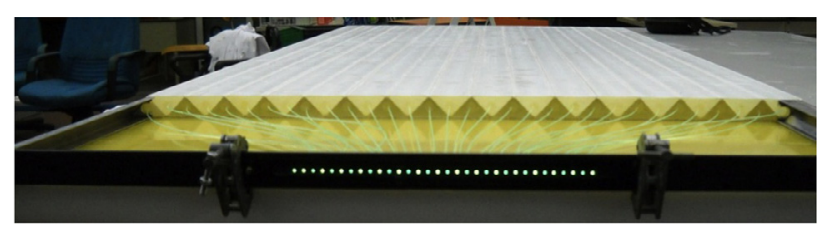

Figure 1. A module with 32 scintillator bars. The wavelength shifting fibers and their connector are visible.

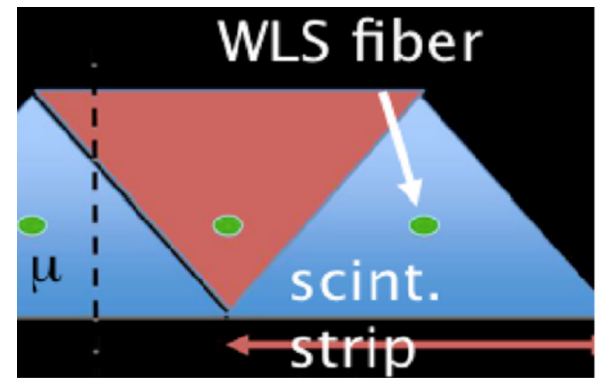

Figure 2. The triangular profile of the scintillators.

environmental light.

The WLS shifting fibres carry the light produced by a particle crossing the scintillators to silicon photomultipliers (SiPM), a recent solid-state technology that in a number of applications is replacing the photo-multiplier tubes. Their low power consumption (less than $1 \mathrm{~mW}$ per channel) is an important feature for applications on sites where an electricity supply is not available. On the other hand, the temperature dependence of their gain requires special attention to monitor and control the SiPMs temperature by a cooling system.

At the Puy de Dôme, the front-end electronics of each module was based on the ASIC chip SPIROC, especially developed for SiPMs by the OMEGA group at LAL, CNRS-IN2P3 (http: / / omega.in2p3.fr). In later developments, the SPIROC chip has been upgraded to the EASIROC chip. The chip has 32 channels, the correct number to equip one module. It is hosted on a hybrid printed circuit board, where the electro-optical connector and the data acquisition electronics of the module are also located. The front-end board ("slave" boards) of each module generates an OR signal from its 32 bars (OR32), which triggers local data acquisition and is sent to a "master" board. On the basis of the OR32 from all modules, the master board generates a trigger that is sent to the slaves for data transmission to the master itself. A detector "plane" consists of two adjacent modules, with 64 scintillator bars in total that are read out by two independent front-end boards. It has an area of about $1 \times 1 \mathrm{~m}^{2}$ area and gives one of the two coordinates ( $\mathrm{X}$ or $\mathrm{Y}$ ) of the impact point of the muon. Two planes with orthogonal bars form an X-Y station. The muon telescope at the Puy de Dôme consisted of three X-Y stations, assembled on an aluminium alloy support frame (Figure 3). A $3 \mathrm{~cm}$ thick iron plate, was inserted near the central station, as a pre-shower to identify and reject electrons. The support frame can be rotated in the horizontal plane, in particular to acquire data with the muon telescope pointing to the free sky for calibration purposes.

The muon trajectory is reconstructed by a linear fit of the X-Y-Z positions of the hits in the three stations, where $Z$ is the longitudinal coordinate. The tracking redundancy provided by three stations rejects 


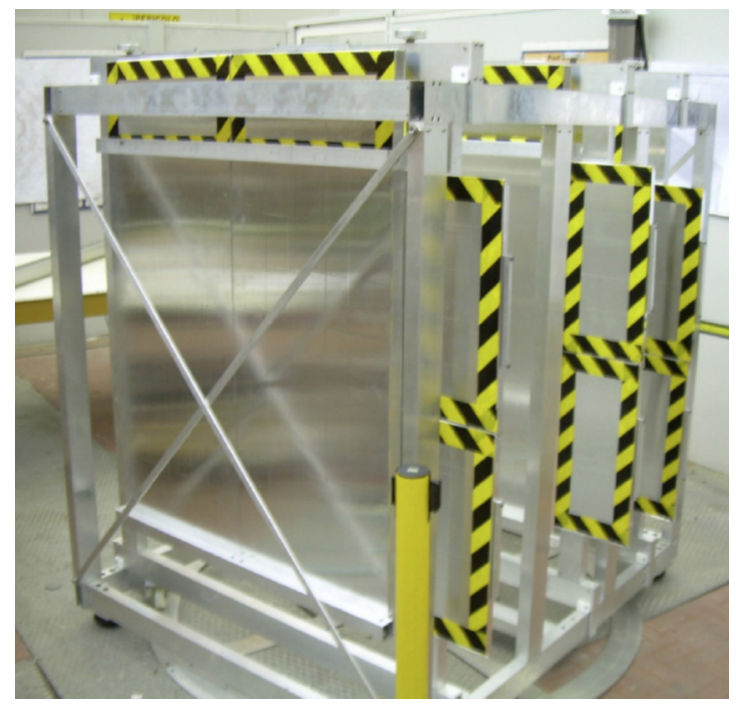

Figure 3. The detector frame with the three $\mathrm{X}-\mathrm{Y}$ planes mounted. The frame can be oriented using the rotating platform visible at the bottom.

the background induced by cosmic-ray showers simultaneously hitting the three stations. The three stations also reduce the probability of accidental triggers produced by thermal noise in the SiPMs. In the future, a further background rejection will come from a timeof-flight measurement to discriminate muons coming from the opposite direction. The background from stray particles of low energy coming from the correct direction is less straightforward to handle. Its estimate is among the aims of the measurement campaign at the Puy de Dôme.

\section{Muon track reconstruction}

The first step in muon track reconstruction is performed independently for each detector module and consists in clustering hits above threshold. The aim is the reconstruction of the coordinates corresponding to muon trajectories, as input to the following step that consists in the reconstruction of the muon tracks.

Among advantages, the use of a solid-state technology as the SiPMs implies handling the dark rate from thermal noise. For this purpose, a calibration of each channel is periodically performed. All channels are acquired randomly, giving the distribution of their electronic pedestal and the dark noise. The distribution of the pedestals of each channel has a peak with specific width and amplitude, which depend on the entire electronic chain. A signal from a SiPM is selected for further analysis if it is at least $5 \mathrm{rms}$ above the pedestal amplitude.

Adjacent bars produce a cluster with size equal to their number. Each cluster is characterized by its position (average of the positions of the corresponding bars), by the number of its bars and by the cluster size, i.e. by the total number of rms from the pedestal am- plitude of its strips. The latter is related to the light release in the bars. The clusters are sorted in descending order according to their size.

In the following step, the X-Z and Y-Z track projections are reconstructed independently. All possible combinations of clusters in the external planes are considered, starting from the clusters with the largest size. If a cluster in the inner plane is found in the neighbourhood of the predicted track position, a linear fit of three clusters is performed and a track projection candidate is obtained. In case of more than one track, the best track is selected as that with the highest size of the smallest cluster (in rms units).

The pair of the best tracks in the X-Z and Y-Z projections forms a three-dimensional track. The efficiency of the reconstruction and tracking algorithm is $92 \%$. Further cuts are applied to improve the quality of the selected sample. The values of the quality cuts were obtained, using the Punzi estimator [Punzi 2003], giving maximum the reconstruction and tracking efficiency on muon sample, and minimum on random triggers sample. As quality cuts, the $\chi^{2}$ of the tracks fits in each projection are required to be less than 5 , the size of minimum cluster of the track is required to be in the range 20-120 (in rms unit) and the number of bars of clusters of the track is required to be less than 5 .

A further cut - the isolation cut - is applied to select muons and reject spurious triggers or other particles, e.g. electrons. The isolation cut requires that no further cluster is present in the detector together with the best track candidate in the event. This sample of "golden tracks" amounts to about $47 \%$ of the triggered events.

\section{Data taking}

The measurement campaign the Puy de Dôme lasted 92 days in real time from June to December 2013. Accounting for the duty cycle, they correspond to about 80 effective days. About 27.5 million events were acquired. The first part of the measurement campaign was dedicated to the calibration of the detectors on site, integrating approximately 3.5 million events pointing to the free sky. The golden track sub-sample consists of about 11.4 million and 1.7 million respectively.

During the data taking the detector was constantly monitored by taking pedestal runs and by measurements of the rates of all channels. The monitoring reduced the duty cycle to about $80 \%$. The trigger rate (the coincidence of the six single view planes) had an average value of $3.6 \mathrm{~Hz}$ and $4 \mathrm{~Hz}$ with the telescope pointing towards the Puy de Dôme and towards the free sky for calibration runs, respectively. The selection criteria reduce the rate to about $2 \mathrm{~Hz}$ for golden tracks.

Figure 4 shows that the golden track rate, the trig- 


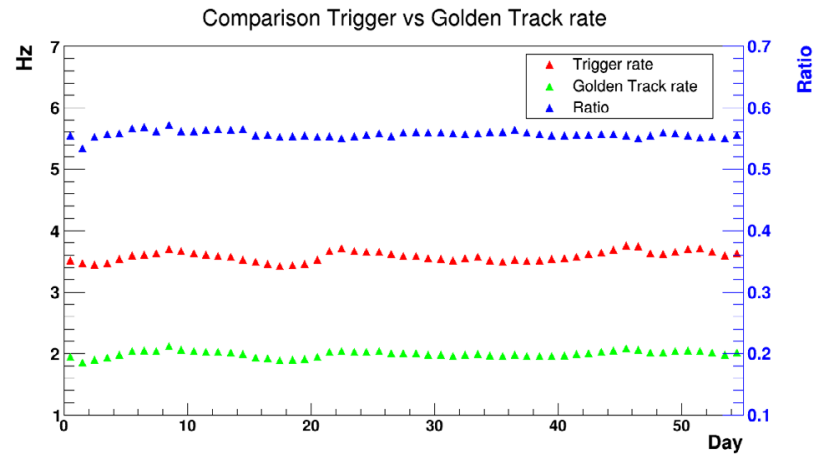

Figure 4. Golden track rate (red dots), trigger rate (green dots) and their ratio (blue dots and scale) over the data taking period.

ger rate and their ratio over the effective days of data taking period, corrected for the dead time (20\%). Some fluctuations are observed in the trigger rate, but the stability of the fraction of golden tracks indicates that shows that the data sample is homogeneous.

In spite of the fact that the temperature of the SiPMs was maintained constant by a cooling system, some fluctuation was observed in the OR32 rate. This fluctuation was correlated (Figure 5) to the temperature of the laboratory housing the detector, which was monitored and had fluctuations of about $3^{\circ} \mathrm{C}$. Indeed,

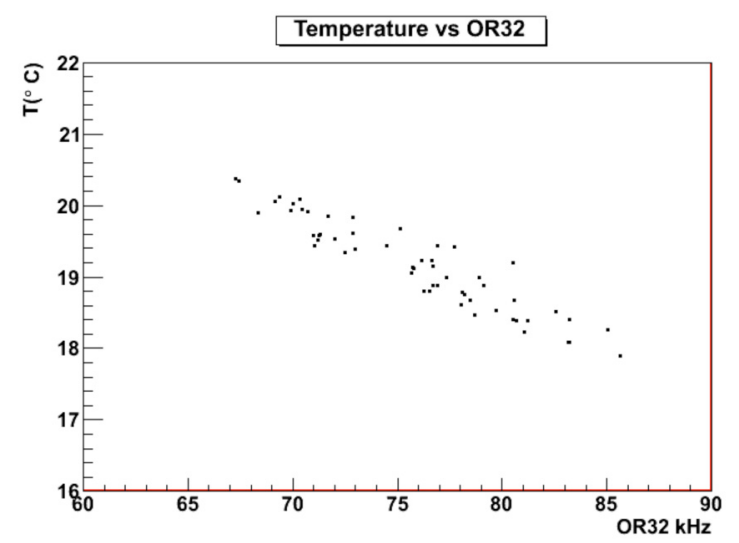

Figure 5. Correlation of the front-end board temperature with the OR32 rate.

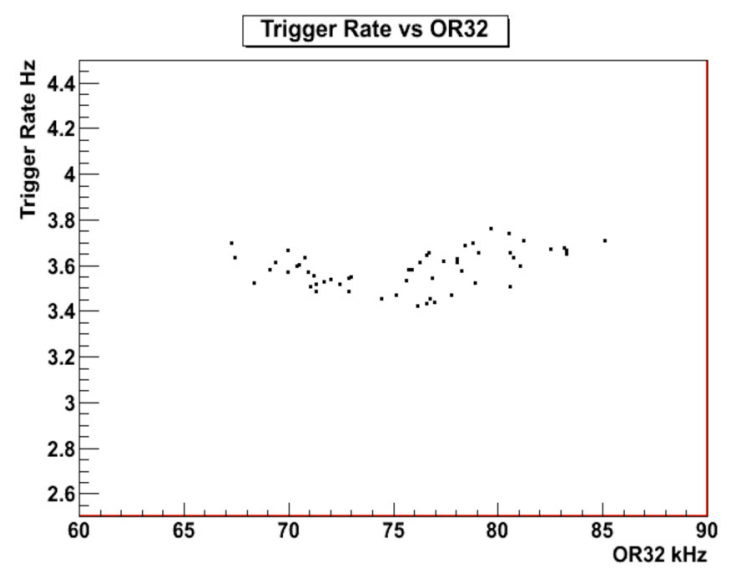

Figure 6. Trigger rate versus fluctuations of the OR32 rate.

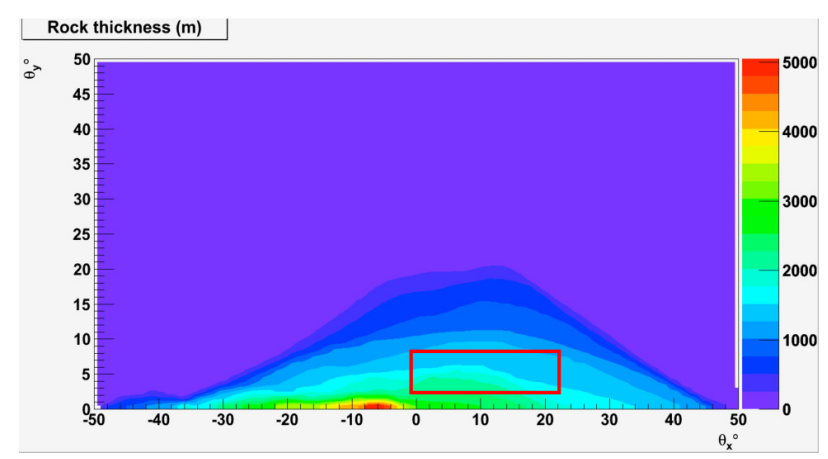

Figure 7. Puy de Dôme rock thickness. The red rectangle indicates the control region.

the front-end electronic boards followed the room temperature. However, the fluctuations in the OR32 rate were not reflected on the rate of their coincidences required by the trigger (Figure 6).

\section{Results}

Future applications of muography require the capability of carrying out investigations over rock thickness larger of $1 \mathrm{~km}$. The evaluation and the study of the background to be overcome was one of the aims of the measurement campaign at Puy de Dôme, with the purpose of having indications about possible upgrades. We thus selected a "control region" with a average rock thickness about $1.5 \mathrm{~km}$ as seen by the muon telescope (Figure 7) and in this region we compared the measured to the expected muon transmission. The muon transmission is defined as the ratio of the muon
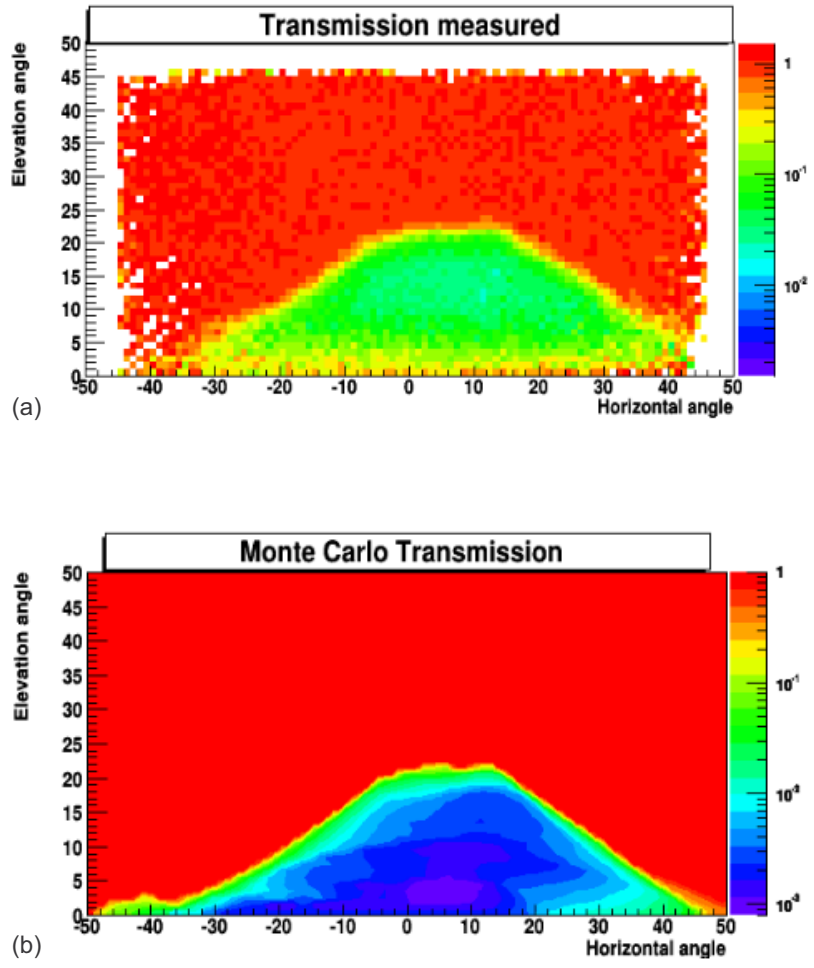

Figure 8. Measured transmission (a) and expected transmission (b). 


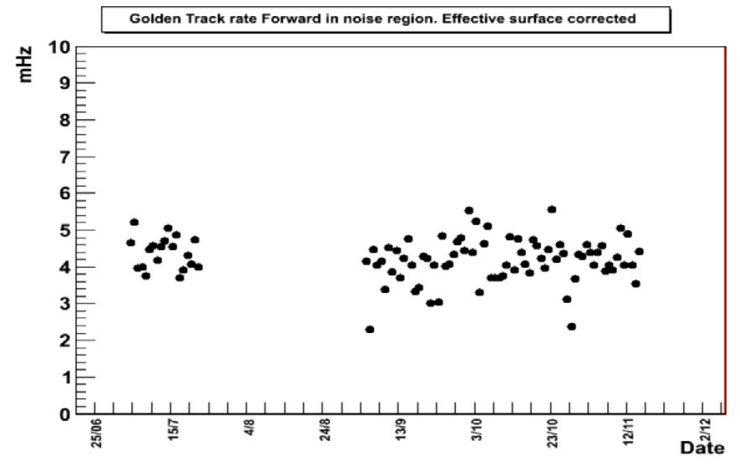

Figure 9. Measured golden track rate per day in the control region.

flux crossing the volcano to the muon flux incident on the volcano itself.

The incident flux can be estimated by a Monte Carlo simulation based on the spectra available from the literature, or can be obtained by taking data with the telescope directed towards the open sky. The latter method has the advantage that the efficiency and the geometrical acceptance of the detector cancel out in the transmission measurement, as they equally affect both the measured and the free sky flux.

Figure 8 shows the measures and the expected trasmission over the whole mountain. In the control area the measured rate is about $4.5 \mathrm{mHz}$ (Figure 9), to be compared to an expected rate of $0.078 \mathrm{mHz}$. The measured rate is two order of magnitude higher than expected.

\section{Conclusion}

The measurement campaign at the Puy de Dôme showed that the MURAY detector prototype acquired stably for a period of about 3 months. The measurements indicate that for a rock thickness larger than $1 \mathrm{~km}$ a substantial background affects the data. This background has physical origin and, probably, consists of low energy muons that are scattered in the rock deviating from their initial direction or in general stray particles. These muons cross the detector and mimic muons from different parts of the mountain, producing a uniform residual background.

Additional tools for background rejection are foreseen, starting from a the time-of-flight measurement. The insertion of additional layers of absorbers will be studied and optimized for the rejection of stray particles.

\section{References}

Alvarez, L.W., J.A. Anderson, F. El Bedwei, J. Burkhard, A. Fakhry, A. Girgis, A. Goneid, F. Hassan, D. Iverson, G. Lynch, Z. Miligy, A.H. Moussa, MohammedSharkawi and L. Yazolino (1970). Search for Hidden Chambers in the Pyramids, Science, 167, 832-839. Ambrosino, F., et al. (2014). The MU-RAY project: de- tector technology and first data from Mt. Vesuvius, J. Instrum., 9, C02029.

Baringer, P., et al. (2001). Cosmic-ray tests of the DØ preshower detector, Nucl. Instrum. Meth. A, 469, 295-310.

Cârloganu, C., et al. (2013). Towards a muon radiography of the Puy de Dôme, Geosci. Instrum. Meth., 2 (1), 55-60.

Punzi, G. (2003). Sensitivity of Searches for New Signals and Its Optimization, PHYSTAT2003 (SLAC, Stanford, California, September 8-11, 2003), 79-83.

Tanaka, H.K.M., T. Nakano, S. Takahashi, J. Yoshida and K. Niwa (2007). Development of an emulsion imaging system for cosmic-ray muon radiography to explore the internal structure of a volcano, Mt. Asama, Nucl. Instrum. Meth. A, 575, 489-497.
${ }^{\star}$ Corresponding author: Pasquale Noli, Istituto Nazionale di Fisica Nucleare (INFN), Sezione di Napoli, Naples, Italy; email: noli@na.infn.it.

C 2017 by the Istituto Nazionale di Geofisica e Vulcanologia. All rights reserved. 